\title{
A BOUNDARY HARNACK INEQUALITY FOR SINGULAR EQUATIONS OF $p$-PARABOLIC TYPE
}

\author{
TUOMO KUUSI, GIUSEPPE MINGIONE, AND KAJ NYSTRÖM
}

(Communicated by Tatiana Toro)

\begin{abstract}
We prove a boundary Harnack type inequality for nonnegative solutions to singular equations of $p$-parabolic type, $2 n /(n+1)<p<2$, in a time-independent cylinder whose base is $C^{1,1}$-regular. Simple examples show, using the corresponding estimates valid for the heat equation as a point of reference, that this type of inequality cannot, in general, be expected to hold in the degenerate case $(2<p<\infty)$.
\end{abstract}

\section{INTRODUCTION AND RESULTS}

In recent years the study of boundary estimates, and boundary Harnack inequalities in particular, for $p$-harmonic functions, $p \neq 2,1<p<\infty$, in Lipschitz domains and in domains which are well approximated by Lipschitz domains in the Hausdorff distance sense have been advanced; see [18]-21]. These estimates have subsequently been used (see [22]-24]) to solve several problems concerning regularity and free boundary regularity for the $p$-Laplace operator. In this note we initiate the study of the corresponding parabolic theory. In particular, we consider boundary estimates involving quasilinear parabolic operators of the type

$$
H u:=u_{t}-\operatorname{div} a(D u),
$$

in time-independent domains of the form $\mathcal{O}=\Omega \times(0, T) \subset \mathbb{R}^{n} \times \mathbb{R}$, where $\Omega \subset \mathbb{R}^{n}$ is a bounded domain and $n \geq 2$. The vector field $a: \mathbb{R}^{n} \rightarrow \mathbb{R}^{n}$ is assumed to be $C^{1}$-regular and is assumed to satisfy

$$
\left\{\begin{array}{c}
|a(\eta)|+|\partial a(\eta)||\eta| \leq L|\eta|^{p-1} \\
\nu|\eta|^{p-2}|\xi|^{2} \leq\langle\partial a(\eta) \xi, \xi\rangle
\end{array}\right.
$$

whenever $\eta, \xi \in \mathbb{R}^{n}$, for some fixed parameters $0<\nu \leq L$, and where, in general, $p$ is allowed to vary in the range $1<p<\infty$. The prototype for this type of operator is the operator

$$
u \rightarrow u_{t}-\operatorname{div}\left(|D u|^{p-2} D u\right) .
$$

The operator in (1.3) is often called the $p$-parabolic operator or the evolutionary $p$-Laplace operator. It is well known (see [4]) that solutions to the $p$-parabolic equation exhibit quite different behaviors in the parameter regimes $2<p<\infty$ (degenerate case) and $1<p<2$ (singular case). In particular, in the degenerate

Received by the editors August 29, 2012.

2010 Mathematics Subject Classification. Primary 35K10, 35K67, 35K92, 35B65.

Key words and phrases. $p$-parabolic equation, degenerate, singular, Harnack inequality, boundary Harnack inequality, Lipschitz domain $C^{1, \alpha}$-domain, $C^{1,1}$. 
case the phenomenon of finite speed propagation is present, and in the singular case solutions will go extinct. Furthermore, the singular case is often divided into the regimes $2 n /(n+1)<p<2$ (super-critical case) and $1<p \leq 2 n /(n+1)$ (subcritical case), and we will here, in the singular case and due to the lack of theory in the sub-critical case, exclusively consider the super-critical case. We also note that when $p=2$, the evolutionary $p$-Laplace operator coincides with the familiar heat operator.

1.1. Background on the linear theory. To outline the type of results we are aiming at, and to put this ambition into context, here we first briefly discuss the corresponding linear theory and the corresponding estimates for the heat equation $(p=2)$ in the setting of bounded Lipschitz domains. We say that $\Omega \subset \mathbb{R}^{n}$ is a bounded Lipschitz domain if there exists a finite set of (standard Euclidean) balls $\left\{B\left(z_{i}, r_{i}\right)\right\}$, with $z_{i} \in \partial \Omega$ and $r_{i}>0$, such that $\left\{B\left(z_{i}, r_{i}\right)\right\}$ constitutes a covering of an open neighborhood of $\partial \Omega$ and such that, for each $i$,

$$
\begin{aligned}
\Omega \cap B\left(z_{i}, 4 r_{i}\right) & =\left\{x=\left(x^{\prime}, x_{n}\right) \in \mathbb{R}^{n}: x_{n}>\phi_{i}\left(x^{\prime}\right)\right\} \cap B\left(z_{i}, 4 r_{i}\right), \\
\partial \Omega \cap B\left(z_{i}, 4 r_{i}\right) & =\left\{x=\left(x^{\prime}, x_{n}\right) \in \mathbb{R}^{n}: x_{n}=\phi_{i}\left(x^{\prime}\right)\right\} \cap B\left(z_{i}, 4 r_{i}\right),
\end{aligned}
$$

in an appropriate coordinate system and for a Lipschitz function $\phi_{i}$. The Lipschitz constants of $\Omega$ are defined to be $M=\max _{i}\left\|\mid \nabla \phi_{i}\right\|_{\infty}$ and $r_{0}=\min _{i} r_{i}$. Given a bounded Lipschitz domain $\Omega$ with constants $M, r_{0}$, we let, for any $\left(x_{0}, t_{0}\right) \in$ $\partial \Omega \times(0, T)$ and $r<\min \left\{r_{0} / \max \{2,2 M\}, \sqrt{\left(T-t_{0}\right) / 4}, \sqrt{t_{0} / 4}\right\}$,

$$
A_{r}\left(x_{0}, t_{0}\right)=\left(x_{0}+2 M r e_{n}, t_{0}\right), \quad A_{r}^{ \pm}\left(x_{0}, t_{0}\right)=\left(x_{0}+2 M r e_{n}, t_{0} \pm 2 r^{2}\right),
$$

where $e_{n}$ is the unit vector pointing in the positive $x_{n}$-direction and defined through the local coordinate system. Then each of these three points are contained in $\Omega \times(0, T)$ and

$$
c^{-1} r<d_{p}\left(\left(x_{0}, t_{0}\right), P\right)<c r \text { and } d_{p}(P, \partial \Omega \times(0, T)) \geq c^{-1} r,
$$

for some $c=c(n, M), 1 \leq c<\infty$, whenever $P \in\left\{A_{r}\left(x_{0}, t_{0}\right), A_{r}^{+}\left(x_{0}, t_{0}\right), A_{r}^{-}\left(x_{0}, t_{0}\right)\right\}$. Here $d_{p}$ denotes the standard parabolic distance function, i.e., $d_{p}((x, t),(y, s))=$ $\max \left\{|x-y|,|t-s|^{1 / 2}\right\}$ whenever $(x, t),(y, s) \in \mathbb{R}^{n} \times \mathbb{R}$, and $d_{p}((x, t), \partial \Omega \times(0, T))$ is the parabolic distance from $(x, t)$ to $\partial \Omega \times(0, T)$. We let $C_{r}(x, t)=B(x, r) \times$ $\left(t-r^{2}, t+r^{2}\right)$ whenever $(x, t) \in \mathbb{R}^{n+1}$ and $r>0$. Consider now a bounded Lipschitz domain $\Omega$ as above with constants $M$ and $r_{0}$. Furthermore, let $u$ and $v$ be two nonnegative solutions to the heat equation in $(\Omega \times(0, T)) \cap C_{2 r_{0}}\left(x_{0}, t_{0}\right)$ and assume that both $u$ and $v$ vanish continuously on $(\partial \Omega \times(0, T)) \cap C_{2 r_{0}}\left(x_{0}, t_{0}\right)$. The following result is essentially due to [8] (see also [11, [8], 26], [9], 10], 27] and [28] for more): There exist constants $c_{1} \equiv c_{1}\left(n, M, m_{u}^{+} / m_{u}^{-}, m_{v}^{+} / m_{v}^{-}\right), c_{2} \equiv$ $c_{2}(n, M), 1 \leq c_{1}, c_{2}<\infty$, and $\sigma \equiv \sigma\left(n, M, m_{u}^{+} / m_{u}^{-}, m_{v}^{+} / m_{v}^{-}\right), 0<\sigma<1$, where $m_{u}^{ \pm}=u\left(A_{r_{0} / c_{2}}^{ \pm}\left(x_{0}, t_{0}\right)\right), m_{v}^{ \pm}=v\left(A_{r_{0} / c_{2}}^{ \pm}\left(x_{0}, t_{0}\right)\right)$, such that

$$
\left|\frac{u(x, t)}{v(x, t)}-\frac{u(y, s)}{v(y, s)}\right| \leq c_{1} \frac{u\left(A_{r}\left(x_{0}, t_{0}\right)\right)}{v\left(A_{r}\left(x_{0}, t_{0}\right)\right)}\left(\frac{d_{p}((x, t),(y, s))}{r}\right)^{\sigma}
$$

holds whenever $(x, t),(y, s) \in(\Omega \times(0, T)) \cap C_{r / 4}\left(x_{0}, t_{0}\right)$ and $0<r<r_{0} / c_{2}$. An important feature of this result is that the statement is both forward and backward in time - something which initially may seem unnatural for the heat equation considering the time-lag generally appearing in the parabolic Harnack inequality. 
However, the fact that $u$ and $v$ both vanish continuously on a large portion of $(\partial \Omega \times(0, T))$ enables one to establish an elliptic type Harnack inequality for them and subsequently the above result; see [8].

1.2. Degenerate versus singular. As mentioned above it is well known that solutions to the $p$-parabolic equation exhibit quite different behaviors in the degenerate case compared to the singular case. In particular, in the degenerate case the phenomenon of finite speed propagation is present, and due to this simple examples show that one cannot expect the result in Section 1.1 to generalize to the $p$-parabolic equation in the degenerate case without imposing additional conditions on $u$ and $v$. Indeed, simply consider the setting of the half space $\mathbb{R}_{+}^{n+1}:=\mathbb{R}_{+}^{n} \times \mathbb{R}=\left\{\left(x_{1}, \ldots, x_{n}, t\right) \in \mathbb{R}^{n} \times \mathbb{R}: x_{n}>0\right\}$. Since $u=x_{n}$ is a solution to the $p$-parabolic equation in $\mathbb{R}_{+}^{n+1}$, and since $u=x_{n}$ vanishes continuously on the boundary of $\mathbb{R}_{+}^{n+1}$, it is obvious that in the degenerate case two nonnegative solutions to the $p$-parabolic equation in $\mathbb{R}_{+}^{n+1}$ need not have the same decay at the boundary since we could, in general, have a solution which is zero in a neighborhood of the boundary. In this case one could say that the solution decays exponentially at the boundary and there is no chance to control its boundary behavior, from above and below, using the linear function $u=x_{n}$. A slightly more advanced counterexample to a conjecture on linear growth at the boundary of the half space $\mathbb{R}_{+}^{n+1}$ is supplied by the function $v(x, t)=c_{p}(T-t)^{-1 /(p-2)} x_{n}^{p /(p-2)}$, $p>2, T>0$ fixed, for an appropriate constant $c_{p}$. This discussion gives at hand, in particular, that in the degenerate case one cannot in general expect results in the spirit of Section 1.1 unless imposing additional restrictions on the set of functions considered to enforce some initial estimates to proceed from. Currently it is not clear to us what these estimates should be.

The singular case differs considerably from the degenerate case, and we emphasize that here we only consider $p$ in the range

$$
2 n /(n+1)<p<2
$$

to ensure the validity of suitable Harnack inequalities; see 4. In the singular case there is, though solutions may go extinct, a phenomena of infinite (in space) propagation, and in the singular range the equation exhibits elliptic features as seen from the forward-backward Harnack inequality valid for positive solutions to the singular $p$-parabolic equation $(2 n /(n+1)<p<2)$. To recall this important property we let $\mathcal{O}=\Omega \times(0, T)$, where $\Omega \subset \mathbb{R}^{n}$ a bounded domain and $T>0$. Let $p$ as in (1.5) be given and suppose that $u$ is a nonnegative and continuous weak solution to (1.1) in $\mathcal{O},\left(\tilde{x}_{0}, \tilde{t}_{0}\right) \in \mathcal{O}$, and assume that $u\left(\tilde{x}_{0}, \tilde{t}_{0}\right)>0$. The following result has been proved in [7]. There are positive constants $c_{i} \equiv c_{i}(n, p, \nu, L), i \in$ $\{1,2,3\}$, such that if

$$
B\left(\tilde{x}_{0}, 8 r\right) \times\left(\tilde{t}_{0}-c_{1} u\left(\tilde{x}_{0}, \tilde{t}_{0}\right)^{2-p}(8 r)^{p}, \tilde{t}_{0}+c_{1} u\left(\tilde{x}_{0}, \tilde{t}_{0}\right)^{2-p}(8 r)^{p}\right) \Subset \mathcal{O},
$$

then

$$
c_{2}^{-1}\left(\sup _{x \in B\left(\tilde{x}_{0}, r\right)} u\left(x, \tau_{1}\right)\right) \leq u\left(\tilde{x}_{0}, \tilde{t}_{0}\right) \leq c_{2}\left(\inf _{x \in B\left(\tilde{x}_{0}, r\right)} u\left(x, \tau_{2}\right)\right)
$$

for all $\tau_{i}, i \in\{1,2\}$, such that

$$
\tau_{1}, \tau_{2} \in\left(\tilde{t}_{0}-c_{3} u\left(\tilde{x}_{0}, \tilde{t}_{0}\right)^{2-p} r^{p}, \tilde{t}_{0}+c_{3} u\left(\tilde{x}_{0}, \tilde{t}_{0}\right)^{2-p} r^{p}\right) .
$$


Hence, for $p$ as in (1.5), a forward, backward and elliptic Harnack inequality is valid for nonnegative solutions. While this Harnack inequality is still intrinsic, it distinguishes the range in (1.5) from the range $2<p<\infty$, as in the latter case only the standard, but still intrinsic, forward in time Harnack inequality holds; see [6]. Based on this discussion and the simple examples above, we here limit ourselves to singular equations of $p$-parabolic type as defined in (1.1), (1.2), and for $p$ as in (1.5), and our main result is a version of the result in Section 1.1 valid for $p$ in the range $2 n /(n+1)<p<2$ and in the setting of time-independent $C^{1,1}$-regular cylinders. The argument outlined below does not generalize to time-independent Lipschitz or $C^{1}$-regular cylinders, and we hope to develop different arguments to cope with these more challenging situations in future papers.

1.3. Results. We say that $\Omega \subset \mathbb{R}^{n}$ is a bounded $C^{1,1}$-regular domain if there exists a finite set of balls $\left\{B\left(z_{i}, r_{i}\right)\right\}$, with $z_{i} \in \partial \Omega$ and $r_{i}>0$, such that $\left\{B\left(z_{i}, r_{i}\right)\right\}$ constitutes a covering of an open neighborhood of $\partial \Omega$ and such that, for each $i$, (1.4) holds in an appropriate coordinate system and for a $C^{1,1}$-regular function $\phi_{i}$. Since we will prove a result which is local in space, we will in the following, using that the family of operators in (1.1), (1.2) is closed under translations (in space and time) and rotations (in space), simply consider the situation when $0 \in \partial \Omega$ (translation) and $\nabla \phi(0)=0$ (rotation), and

$$
\begin{aligned}
\Omega \cap B\left(0,4 r_{0}\right) & =\left\{x=\left(x^{\prime}, x_{n}\right) \in \mathbb{R}^{n}: x_{n}>\phi\left(x^{\prime}\right)\right\} \cap B\left(0,4 r_{0}\right), \\
\partial \Omega \cap B\left(0,4 r_{0}\right) & =\left\{x=\left(x^{\prime}, x_{n}\right) \in \mathbb{R}^{n}: x_{n}=\phi\left(x^{\prime}\right)\right\} \cap B\left(0,4 r_{0}\right),
\end{aligned}
$$

for some $r_{0}$ fixed and for some $C^{1,1}$-regular function $\phi$. In particular, $\phi(0)=0$ and we consider a fixed coordinate system and the existence of a $C^{1,1}$-regular function $\phi$ defining $\Omega \cap B\left(0,4 r_{0}\right)$ and $\partial \Omega \cap B\left(0,4 r_{0}\right)$. Using this fixed coordinate system we note that we can always, by the assumption that $\phi$ is $C^{1,1}$-regular and $\nabla \phi(0)=0$, find small enough $r_{0}$ such that

$$
\sup _{x^{\prime} \in B^{\prime}\left(0,4 r_{0}\right)}\left|\nabla \phi\left(x^{\prime}\right)\right| \leq 1 .
$$

In (1.10) and in what follows, we let $x^{\prime}=\left(x_{1}, \ldots, x_{n-1}\right)$ whenever $x \in \mathbb{R}^{n}$ and we let $B^{\prime}$ denote the orthogonal projection of $B$ onto $\left\{x=\left(x^{\prime}, x_{n}\right) \in \mathbb{R}^{n}: x_{n}=0\right\}$. Chosen this way, $r_{0}$ depends only on the $C^{1,1}$-norm of $\phi$. Moreover, recall that a bounded domain $\Omega \subset \mathbb{R}^{n}$ is said to satisfy a uniform inner ball condition if there exists $r_{0}, 0<r_{0}<\infty$, such that the following is true. There exists, whenever $x_{0} \in \partial \Omega$, a point $\tilde{x}_{0} \in \Omega$ such that $B\left(\tilde{x}_{0}, r_{0}\right) \subset \Omega$ and $\overline{B\left(\tilde{x}_{0}, r_{0}\right)} \cap \partial \Omega=\left\{x_{0}\right\}$. Similarly, $\Omega \subset \mathbb{R}^{n}$ is said to satisfy a uniform outer ball condition if the above holds with $\Omega$ replaced by the complement of $\Omega$. It is a well known fact (see for example 2] for a proof) that

$$
\text { a bounded domain } \Omega \subset \mathbb{R}^{n} \text { satisfies a uniform inner (outer) }
$$

$$
\text { ball condition for some } r_{0}, 0<r_{0}<\infty \text {, if and only if } \Omega \text { is } C^{1,1} \text {-regular. }
$$

From now on $r_{0}$ is taken to be small enough so that both (1.10) and (1.11) hold. Without loss of generality we may assume that $r_{0} \in(0,1]$.

We need to introduce cubes adapted to $\phi$. Indeed, given a point $\left(x_{0}, t_{0}\right)=$ $\left(x_{0}^{\prime}, \phi\left(x_{0}^{\prime}\right), t_{0}\right) \in \mathbb{R}^{n+1}, r \leq r_{0} /(10 \sqrt{n})$, and $\lambda>0$, we let

$$
\begin{aligned}
Q_{r, \phi}^{\lambda,+}\left(x_{0}, t_{0}\right)= & \left\{(x, t) \in \mathbb{R}^{n+1}:\left|x_{i}-\left(x_{0}\right)_{i}\right|<r \text { for } i \in\{1, \ldots, n-1\},\right. \\
& \left.\phi\left(x^{\prime}\right)<x_{n}<10 r+\phi\left(x^{\prime}\right),\left|t-t_{0}\right|<\lambda^{2-p} r^{p}\right\} .
\end{aligned}
$$


We also let

$$
\begin{gathered}
\Delta_{r, \phi}^{\lambda}\left(x_{0}, t_{0}\right)=\left\{(x, t) \in \mathbb{R}^{n+1}:\left|x_{i}-\left(x_{0}\right)_{i}\right|<r \text { for } i \in\{1, \ldots, n-1\},\right. \\
\left.x_{n}=\phi\left(x^{\prime}\right),\left|t-t_{0}\right|<\lambda^{2-p} r^{p}\right\} .
\end{gathered}
$$

Consider $\mathcal{O}=\Omega \times(0, T)$, where $\Omega \subset \mathbb{R}^{n}$ is as above and $T>0$. Let $\left(\tilde{x}_{0}, \tilde{t}_{0}\right) \in \Omega_{T}$, assume (1.9), and assume that $\tilde{x}_{0} \in \Omega \cap B\left(0, r_{0} /(100 \sqrt{n})\right)$. Let $x_{0}$ denote the projection of $\tilde{x}_{0}$ along $e_{n}$ and onto $\partial \Omega$ and let $r=\left|x_{0}-\tilde{x}_{0}\right|, t_{0}=\tilde{t}_{0}$. Then $x_{0} \in \partial \Omega \cap B\left(0, r_{0} /(50 \sqrt{n})\right)$. Let $u$ and $v$ be two functions which are nonnegative and continuous in a neighborhood of $\left(\tilde{x}_{0}, \tilde{t}_{0}\right)$ and assume that

$$
\theta_{u}:=u\left(\tilde{x}_{0}, \tilde{t}_{0}\right) \text { and } \theta_{v}:=v\left(\tilde{x}_{0}, \tilde{t}_{0}\right) \text { are positive } .
$$

We also assume that

$$
\begin{aligned}
& \theta_{u}^{2-p}(8 r)^{p}<t_{0}<T-\theta_{u}^{2-p}(8 r)^{p} \\
& \theta_{v}^{2-p}(8 r)^{p}<t_{0}<T-\theta_{v}^{2-p}(8 r)^{p} .
\end{aligned}
$$

To formulate our result we will assume the existence of certain intrinsic parameters associated to $u$ and $v$. Indeed, given $u,\left(\tilde{x}_{0}, \tilde{t}_{0}\right)$, and $r$ as above we let $\Gamma_{u}$ denote the set of all values of $\Lambda_{u}, 0<\Lambda_{u}<\infty$, for which the following three restrictions hold. Firstly,

$$
\left(\Lambda_{u}\right)^{2-p}(8 r)^{p}<t_{0}<T-\left(\Lambda_{u}\right)^{2-p}(8 r)^{p} .
$$

Secondly, $u$ is assumed to be a nonnegative solution to (1.1) in $Q_{8 r, \phi}^{\Lambda_{u},+}\left(x_{0}, t_{0}\right)$, continuous on the closure of this set and vanishing continuously on $\Delta_{8 r, \phi}^{\Lambda_{u}}\left(x_{0}, t_{0}\right)$. Thirdly, we assume that

$$
\sup _{Q_{4 r, \phi}^{\Lambda_{u},+}\left(x_{0}, t_{0}\right)} u \leq \Lambda_{u}
$$

We define $\Gamma_{v}$ analogously. Note that assuming (1.15) and using the fact that $\left(\tilde{x}_{0}, \tilde{t}_{0}\right) \in Q_{4 r, \phi}^{\Lambda_{u},+}\left(x_{0}, t_{0}\right),\left(\tilde{x}_{0}, \tilde{t}_{0}\right) \in Q_{4 r, \phi}^{\Lambda_{v},+}\left(x_{0}, t_{0}\right)$, we see that any such $\Lambda_{u}$ and $\Lambda_{v}$ must satisfy $\theta_{u} \leq \Lambda_{u}$ and $\theta_{v} \leq \Lambda_{v}$. In the following we will assume that $u, v$, $\left(\tilde{x}_{0}, \tilde{t}_{0}\right), r, T$ are such that

$$
\text { (1.15) holds and } \Gamma_{u} \neq \emptyset \text { and } \Gamma_{v} \neq \emptyset \text {. }
$$

Based on (1.18), in the following we let $\Lambda_{u}$ and $\Lambda_{v}$ denote the smallest values of $\Lambda_{u}$ and $\Lambda_{v}$ for which (1.17) and the corresponding statement for $\Lambda_{v}$ hold. Note that for these values of $\Lambda_{u}, \Lambda_{v}$, we can assume, without loss of generality, that

$$
\sup _{Q_{4 r, \phi}^{\Lambda_{u},+}\left(x_{0}, t_{0}\right)} u=\Lambda_{u}, \sup _{Q_{4 r, \phi}^{\Lambda_{v},+}\left(x_{0}, t_{0}\right)} v=\Lambda_{v} .
$$

The relevance of this complexity, and of the parameters $\Lambda_{u}, \Lambda_{v}$, is outlined below. In this paper we prove the following theorem.

Theorem 1.1. Let $\mathcal{O}=\Omega \times(0, T)$, where $\Omega \subset \mathbb{R}^{n}$ is a bounded $C^{1,1}$-regular domain and $T>0$. Let $p$ as in (1.5) be fixed, and let $H$ be as in (1.1), (1.2). Let $\left(\tilde{x}_{0}, \tilde{t}_{0}\right),\left(x_{0}, t_{0}\right)$, and $r$ be as above. Let $u$ and $v$ be solutions to (1.1) as stated above, and assume that they satisfy (1.14) and (1.15). Assume further (1.18) and let $\Lambda_{u}$ and $\Lambda_{v}$ be such that (1.19) holds. Assume in addition that there exist $\lambda_{u}, \lambda_{v}$, $1 \leq \lambda_{u}<\infty, 1 \leq \lambda_{v}<\infty$, such that

$$
\theta_{u} \leq \Lambda_{u} \leq \lambda_{u} \theta_{u}, \quad \theta_{v} \leq \Lambda_{v} \leq \lambda_{v} \theta_{v} .
$$


Then there exist constants $c_{1} \equiv c_{1}\left(n, p, \nu, L, r_{0}, \lambda_{u}, \lambda_{v}\right), c_{2} \equiv c_{2}\left(n, p, \nu, L, r_{0}\right), 1 \leq$ $c_{1}, c_{2}<\infty$, and $\sigma \equiv \sigma(n, p, \nu, L), \sigma<1$, such that

$$
\left|\frac{u(x, t)}{v(x, t)}-\frac{u(y, s)}{v(y, s)}\right| \leq c_{1} \frac{\theta_{u}}{\theta_{v}}\left(\frac{|x-y|}{r}+\left(\frac{1}{\theta_{u v}}\right)^{(2 / p-1)}\left(\frac{|s-t|}{r^{p}}\right)^{1 / p}\right)^{\sigma}
$$

holds whenever $(x, t),(y, s) \in Q_{r / c_{2}, \phi}^{\theta_{u v},+}\left(x_{0}, t_{0}\right)$, where $\theta_{u v}=\min \left\{\theta_{u}, \theta_{v}\right\}$.

Note that when $p=2$, then, formally and essentially, Theorem 1.1 coincides with the linear result described in Section 1.1. Indeed, in the case $p=2$ we see that if both $u$ and $v$ vanish on a sufficiently large portion of the lateral boundary, so that we can, following [27], 8], ensure the validity of the forward-backward in time Harnack inequalities

$$
\sup _{Q_{4 r, \phi}^{1,+}\left(x_{0}, t_{0}\right)} u \leq c \theta_{u}, \sup _{Q_{4 r, \phi}^{1,+}\left(x_{0}, t_{0}\right)} v \leq c \theta_{v},
$$

for some $c, 1 \leq c<\infty$, independent of $r$ and $\left(\tilde{x}_{0}, \tilde{t}_{0}\right)$, then

$$
\Lambda_{u}:=\sup _{Q_{4 r, \phi}^{1,+}\left(x_{0}, t_{0}\right)} u \leq c \theta_{u}, \Lambda_{v}:=\sup _{Q_{4 r, \phi}^{1,+}\left(x_{0}, t_{0}\right)} v \leq c \theta_{v} .
$$

Hence, formally the statement of Theorem 1.1 reduces to

$$
\left|\frac{u(x, t)}{v(x, t)}-\frac{u(y, s)}{v(y, s)}\right| \leq c_{1} \frac{u\left(\tilde{x}_{0}, \tilde{t}_{0}\right)}{v\left(\tilde{x}_{0}, \tilde{t}_{0}\right)}\left(\frac{d_{p}((x, t),(y, s))}{r}\right)^{\sigma}
$$

whenever $(x, t),(y, s) \in Q_{r / c_{2}, \phi}^{1,+}\left(x_{0}, t_{0}\right)$. We emphasize that in Section 1.1 the involved dependence of the constants described enters through the constant $c$ in (1.21). We also note that while, formally and when $p=2$, the statement of Theorem 1.1 reduces to a form similar to that in Section 1.1 we are not saying that the constants of Theorem 1.1 by necessity are stable as $p \rightarrow 2$.

1.4. Intrinsic scaling parameters. A crucial ingredient in the regularity theory for the operator in (1.3) is the use of DiBenedetto's intrinsic geometry when deriving local estimates. This amounts to the use of cylinders whose size depends on the solution itself. This is necessary since operators such as the one considered in (1.3) show a strong anisotropy when $p \neq 2$, as the multiplication of a solution to the associated equation by a constant does not yield a solution to a similar equation. One consequence of this is the lack of homogeneous a priori estimates and hence the impossibility to use such estimates in iterative schemes in line with the standard regularity techniques. Instead, the lack of homogeneity must be locally corrected by using scaling parameters and intrinsic geometries, and a key insight from regularity theory (see [1, 4, 5, 14, 17]) is that in general the type of cylinders used must depend on the type of problem/regularity one is currently considering/using. In the context of Theorem 1.1 we see that $\Lambda_{u}, \Lambda_{v}, \lambda_{u}$, and $\lambda_{v}$ serve as intrinsic (scaling) parameters. Indeed, concerning the conditions in (1.19) we note, focusing on $u$, assuming $\left(x_{0}, t_{0}\right)=(0,0), r=1,\left(\tilde{x}_{0}, \tilde{t}_{0}\right)=\left(e_{n}, 0\right)$, that if we define $\tilde{u}(x, t)=u\left(x, t \Lambda_{u}^{2-p}\right) / \Lambda_{u}$ for $(x, t) \in Q_{4, \phi}^{1,+}(0,0)$, then, by construction,

$$
\sup _{Q_{4, \phi}^{1,+}(0,0)} \tilde{u}=1 .
$$


In particular, in this way we can simultaneously normalize the scale to a unit scale and the supremum of the function on the unit scale to 1 . This enables us to ensure the validity of estimates for the gradient of $\tilde{u}$ with constants depending only on $n, p, \nu, L$. Furthermore, the parameter $\lambda_{u}$ ensures a relation between the largest value of $u$ on the large box $Q_{4 r, \phi}^{\Lambda_{u},+}\left(x_{0}, t_{0}\right)$ and the value of $u$ at $\left(\tilde{x}_{0}, \tilde{t}_{0}\right), \theta_{u}$. This relation cannot in general be expected to hold, due to the phenomena of extinction present in the singular case, for some uniform $\lambda$ not depending on $u$. Indeed, $u$ could very well go extinct at $t=\tilde{t}_{0}+\varepsilon$ while $\theta_{u} \neq 0$. Restarting the Cauchy-Dirichlet problem at $t=\tilde{t}_{0}+2 \varepsilon$ by enforcing large positive data on parts of the lateral side of the cylinder from $t=\tilde{t}_{0}+2 \varepsilon$ and putting zero data on the base of the cylinder at $t=\tilde{t}_{0}+2 \varepsilon$, we can construct a solution $u$ such that the supremum of $u$ is large while $\theta_{u}$ is very small, resulting in a very large value of $\lambda_{u}$. This question is related to the possibility of establishing Carleson type estimates (see [27]) for nonnegative solutions to the $p$-parabolic equation. For an account of this type of estimate, for the $p$-parabolic equation and for the porous medium equation, we refer to $[3]$. The condition $\theta_{u} \leq \Lambda_{u} \leq \lambda_{u} \theta_{u}$ also allows us to construct elliptic type Harnack chains, depending on $\lambda_{u}$, to compare values of $u$ close to the boundary and as outlined in the bulk of the paper. In particular, after appropriate normalizations, using (1.20), the construction of elliptic Harnack chains becomes analogous to the construction of Harnack chains for the Laplace operator; see [12.

\section{Preliminaries}

2.1. Weak solution and the Dirichlet problem. If $U \subset \mathbb{R}^{n}$ is open and $1 \leq$ $q \leq \infty$, then by $W^{1, q}(U)$ we denote the space of equivalence classes of functions $f$ with distributional gradient $D f=\left(f_{x_{1}}, \ldots, f_{x_{n}}\right)$, both of which are $q$-th power integrable on $U$. Let

$$
\|f\|_{W^{1, q}(U)}=\|f\|_{L^{q}(U)}+\||D f|\|_{L^{q}(U)}
$$

be the norm in $W^{1, q}(U)$, where $\|\cdot\|_{L^{q}(U)}$ denotes the usual Lebesgue $q$-norm in $U$. Given $t_{1}<t_{2}$ we denote by $L^{q}\left(t_{1}, t_{2}, W^{1, q}(U)\right)$ the space of functions such that for almost every $t, t_{1}<t<t_{2}$, the function $x \rightarrow u(x, t)$ belongs to $W^{1, q}(U)$ and

$$
\|u\|_{L^{q}\left(t_{1}, t_{2}, W^{1, q}(U)\right)}:=\left(\int_{t_{1}}^{t_{2}} \int_{U}\left(|u(x, t)|^{q}+|D u(x, t)|^{q}\right) d x d t\right)^{1 / q}<\infty .
$$

In the following we first describe the concept of weak solutions to

$$
H u=u_{t}-\operatorname{div} a(D u)=0
$$

when the underlying domain considered is not necessarily a cylinder.

Definition 2.1. Let $H$ be as in (2.1) and assume (1.2). We say that a function $u$ is a weak supersolution (subsolution) to (2.1) in an open set $\Xi \Subset \mathbb{R}^{n+1}$ if, whenever $\Xi^{\prime}=U \times\left(t_{1}, t_{2}\right) \Subset \Xi$ with $U \subset \mathbb{R}^{n}$ and $t_{1}<t_{2}$, then $u \in L^{p}\left(t_{1}, t_{2} ; W^{1, p}(U)\right)$ and

$$
\int_{\Xi^{\prime}}\left(\langle a(D u), D \phi\rangle-u \phi_{t}\right) d x d t \geq(\leq) 0
$$

for all nonnegative $\phi \in C_{0}^{\infty}\left(\Xi^{\prime}\right)$. A weak solution is a distributional solution satisfying (2.2) with equality and without sign restrictions for test functions. 
Note, in particular, that in Definition 2.1 no assumption on the time derivative of $u$ is made. Note also that by parabolic regularity theory (see [4]) solutions are locally Hölder continuous after a redefinition on a set of measure zero. In particular, we can in the following assume that any solution $u$ is continuous. Furthermore, we note that it is well known that if, for example, $\Omega \subset \mathbb{R}^{n}$ is a bounded $C^{1,1}$-regular domain, then the cylinder $\mathcal{O}$ is regular for the Dirichlet problem for the operators in (1.1), (1.2); see [13]. In particular, given continuous boundary data $b: \overline{\mathcal{O}} \rightarrow \mathbb{R}$ the problem

$$
\begin{cases}H u=0 & \text { in } \mathcal{O}, \\ u=b & \text { on } \partial_{\mathrm{P}} \mathcal{O}\end{cases}
$$

has a unique weak solution which is continuous on the closure on $\mathcal{O}$. Here $\partial_{\mathrm{P}} \mathcal{O}:=$ $(\bar{\Omega} \times\{0\}) \cup(\partial \Omega \times[0, T])$ denotes the parabolic boundary of $\mathcal{O}$.

2.2. Scaling of solutions. Here we describe scaling properties of (weak) solutions to the equation (2.1). Given $r, \lambda>0$, we consider the cube

$$
\begin{aligned}
Q_{r}^{\lambda}= & \left\{(x, t) \in \mathbb{R}^{n+1}:\left|x_{i}\right|<r \text { for } i \in\{1, \ldots, n-1\},\right. \\
& \left.\left|x_{n}\right|<10 r,|t|<\lambda^{2-p} r^{p}\right\},
\end{aligned}
$$

and given $\left(\tilde{x}_{0}, \tilde{t}_{0}\right) \in \mathbb{R}^{n+1}$ we let $Q_{r}^{\lambda}\left(\tilde{x}_{0}, \tilde{t}_{0}\right)$ be the cube $Q_{r}^{\lambda}$ translated to the point $\left(\tilde{x}_{0}, \tilde{t}_{0}\right)$. Given $\left(\tilde{x}_{0}, \tilde{t}_{0}\right) \in \mathbb{R}^{n+1}, r \leq R, \lambda>0$, suppose that $u$ solves (2.1) in $Q_{R}^{\lambda}\left(\tilde{x}_{0}, \tilde{t}_{0}\right)$. Define

$$
\begin{aligned}
& \tilde{u}(x, t):=\frac{u\left(\tilde{x}_{0}+r x, \tilde{t}_{0}+\lambda^{2-p} r^{p} t\right)}{\lambda}, \\
& \tilde{a}(\eta):=\frac{a((\lambda / r) \eta)}{(\lambda / r)^{p-1}}, \quad \eta \in \mathbb{R}^{n} .
\end{aligned}
$$

Then $\tilde{u}$ solves the equation $\tilde{H} \tilde{u}:=\tilde{u}_{t}-\operatorname{div} \tilde{a}(D \tilde{u})=0$ in $Q_{R / r}^{1}$. In particular, in the case $r=R$, we have that $\tilde{w}$ is a solution in $Q_{1}^{1}$. The new vector field $\tilde{a}(\cdot)$ satisfies bounds

$$
\left\{\begin{array}{c}
|\tilde{a}(\eta)|+|\partial \tilde{a}(\eta)||\eta| \leq L|\eta|^{(p-1)} \\
\nu|\eta|^{p-2}|\xi|^{2} \leq\langle\partial \tilde{a}(\eta) \xi, \xi\rangle
\end{array}\right.
$$

for all $\eta, \xi \in \mathbb{R}^{n}$. In particular, we remark that the assumptions in (1.2) imply the existence of $\bar{\nu} \in(0,1)$, and $c, \bar{L} \geq 1$, depending on $n, p, \nu, L$, such that the following growth and coercivity assumptions hold for every choice $\eta \in \mathbb{R}^{n}$ :

$$
|\tilde{a}(\eta)| \leq \bar{L}|\eta|^{(p-1)}, \quad\langle\tilde{a}(\eta), \eta\rangle \geq \bar{\nu}|\eta|^{p} .
$$

2.3. Gradient estimates. We note that in the proof of Theorem 1.1 we can without loss of generality assume that

$$
\left(x_{0}, t_{0}\right)=(0,0), \quad r=1, \quad\left(\tilde{x}_{0}, \tilde{t}_{0}\right)=\left(e_{n}, 0\right) .
$$

Here we formulate, assuming (2.9), a boundary gradient estimate to be used in the proof of Theorem 1.1.

Lemma 2.2. Let $u$ be as in Theorem 1.1 and assume also that (2.9) holds. Let $\tilde{u}(x, t)=u\left(x, t \Lambda_{u}^{2-p}\right) / \Lambda_{u}$ for $(x, t) \in Q_{4, \phi}^{1,+}(0,0)$ so that, by construction,

$$
\sup _{Q_{4, \phi}^{1,+}(0,0)} \tilde{u}=1 \text {. }
$$


Then Dũ exists and is continuous up to $\Delta_{2, \phi}^{1}(0,0)$ and there exist constants $c \equiv$ $c(n, p, \nu, L), 1 \leq c<\infty$, and $\sigma \equiv \sigma(n, p, \nu, L), 0<\sigma \leq 1$, such that

$$
|D u(x, t)| \leq c, \quad|D \tilde{u}(x, t)-D \tilde{u}(y, s)| \leq c\left(|x-y|+|t-s|^{1 / p}\right)^{\sigma}
$$

hold whenever $(x, t),(y, s) \in Q_{1, \phi}^{1,+}(0,0)$.

Proof. This is a special case of Theorem 0.1 in [25].

\section{Proof of Theorem 1.1}

To prove Theorem 1.1 we first use a barrier type argument to establish linear growth estimates at the boundary, and here the assumption that $\Omega \subset \mathbb{R}^{n}$ is a bounded $C^{1,1}$. regular domain is important. Our proof of Theorem 1.1 is based on the following lemma. In the formulation $r_{0}$ is as described in Section 1.3.

Lemma 3.1. Let $u$ be as in Theorem 1.1 and assume also that (2.9) holds. Then there exist constants $c \equiv c\left(n, p, \nu, L, r_{0}\right), 1 \leq c<\infty$, and $\varrho_{0} \equiv \varrho_{0}(n, p, \nu, L)$, $0<\varrho_{0}<1$, such that

$$
c^{-1} \theta_{u} \cdot\left[x_{n}-\phi\left(x^{\prime}\right)\right] \leq u(x, t) \leq c \lambda_{u} \theta_{u} \cdot\left[x_{n}-\phi\left(x^{\prime}\right)\right]
$$

holds whenever $(x, t) \in\left(\Omega \cap\left(B^{\prime}\left(0, r_{0}\right) \times[-1,1]\right)\right) \times\left(-\theta_{u}^{2-p} \varrho_{0}^{p}, \theta_{u}^{2-p} \varrho_{0}^{p}\right)$.

Proof. First we notice that if $x \in \Omega \cap B\left(0, r_{0}\right)$ and $\hat{x} \in \partial \Omega$ is such that $|x-\hat{x}|=$ $\operatorname{dist}(x, \partial \Omega)$, then

$$
\operatorname{dist}(x, \partial \Omega) \leq\left|x_{n}-\phi\left(x^{\prime}\right)\right| \leq\left|x_{n}-\hat{x}_{n}\right|+\left|\phi\left(\hat{x}^{\prime}\right)-\phi\left(x^{\prime}\right)\right| \leq 2 \operatorname{dist}(x, \partial \Omega)
$$

holds by (1.10) since $\hat{x}_{n}=\phi\left(\hat{x}^{\prime}\right)$. Thus it is enough to prove (3.1) with $x_{n}-\phi\left(x^{\prime}\right)$ replaced with $\operatorname{dist}(x, \partial \Omega)$.

Focusing on the proof of the inequality on the right hand side in (3.1) we consider $\tilde{u}(x, t)=u\left(x, t \Lambda_{u}^{2-p}\right) / \Lambda_{u}$ for all $(x, t)$ such that $\left(x, t \Lambda_{u}^{2-p}\right) \in Q_{4, \phi}^{\Lambda_{u},+}(0,0)$, i.e., for $(x, t) \in Q_{4, \phi}^{1,+}(0,0)$. Note that by construction

$$
\sup _{Q_{4, \phi}^{1,+}(0,0)} \tilde{u}=1 .
$$

In accordance to (1.11) in the following we let $\delta \in\left(0, r_{0} / 10\right]$ and we let

$$
\Omega_{\delta}:=\Omega \cap(-2,2)^{n} \cap\{x \in \Omega: \operatorname{dist}(x, \partial \Omega) \leq \delta\},
$$

where $(-2,2)^{n}$ denotes the $n$-dimensional cube $(-2,2) \times \cdots \times(-2,2)$. Consider then $(z, \tau) \in \Omega_{\delta} \times(-3,3) \subset Q_{4, \phi}^{1,+}(0,0)$. Given $z$, let $\hat{z} \in \partial \Omega$ be such that $|z-\hat{z}|=$ $\operatorname{dist}(z, \partial \Omega)<\delta$, and for this $\hat{z} \in \partial \Omega$ we use (1.11) and let $\hat{z}_{r_{0}} \in \mathbb{R}^{n} \backslash \bar{\Omega}$ be such that $B\left(\hat{z}_{r_{0}}, r_{0}\right) \subset\left(\mathbb{R}^{n} \backslash \bar{\Omega}\right)$ and $\overline{B\left(\hat{z}_{r_{0}}, r_{0}\right)} \cap \partial \Omega=\{\hat{z}\}$. Moreover, we set $I(\tau)=(\tau-1, \tau]$. Focusing on the domain $\Omega_{\delta} \times I(\tau)$, considering $(z, \tau) \in \Omega_{\delta} \times I(\tau)$ fixed, we construct, following the construction in [29, display (4.7)], a barrier as follows. We let

$$
\begin{aligned}
& \psi(x, t):=C(1-\eta(x, t)), \\
& \eta(x, t):=\exp \left(-\delta^{-1}\left(\left|x-\hat{z}_{r_{0}}\right|-r_{0}\right)\right) \exp (t-\tau),
\end{aligned}
$$

where $C$ shall be fixed shortly. Consider the set

$$
D_{\delta}:=\left\{x \in \Omega:\left|x-\hat{z}_{r_{0}}\right|-r_{0}<\delta\right\} \times I(\tau) .
$$

If $(x, t) \in D_{\delta}$, then there is $y \in \partial \Omega$ such that $\left|x-\hat{z}_{r_{0}}\right|=|x-y|+\left|y-\hat{z}_{r_{0}}\right|$ and

$$
\delta+r_{0}>\left|x-\hat{z}_{r_{0}}\right|=|x-y|+\left|y-\hat{z}_{r_{0}}\right| \geq|x-y|+r_{0},
$$


and thus $D_{\delta} \subset \Omega_{\delta} \times I(\tau)$. Moreover, $(z, \tau) \in D_{\delta}$. We now want to prove that $\delta=\delta\left(p, \nu, L, r_{0}\right), 0<\delta \ll 1$, can be chosen so that $\psi$ satisfies

$$
\tilde{u}(x, t) \leq \psi(x, t) \text { whenever }(x, t) \in D_{\delta} .
$$

Using the comparison principle we see that to achieve this it is enough to prove

$$
\begin{array}{ll}
\tilde{u}(x, t) \leq \psi(x, t) & \text { on } \partial_{P} D_{\delta}, \\
\partial_{t} \psi-\operatorname{div} a(D \psi) \geq 0 & \text { in } D_{\delta} .
\end{array}
$$

Appealing to (3.2) and the fact that $\tilde{u}=0$ on $\partial \Omega \times(-4,4)$, it is easy to see that (3.7) is verified if we let $C=(1-\exp (-1))^{-1}$. Hence we focus on (3.8) and we first note that

$$
\partial_{t} \psi-\operatorname{div} a(D \psi)=\partial_{t} \psi-\partial_{\eta_{j}} a_{i}(D \psi) \partial_{x_{i} x_{j}} \psi
$$

and

$$
\partial_{t} \psi=-C \eta, \quad D \psi=\delta^{-1} C \eta \frac{x-\hat{z}_{r_{0}}}{\left|x-\hat{z}_{r_{0}}\right|} .
$$

Furthermore, we have that

$$
\begin{aligned}
(C \eta)^{-1} \partial_{x_{i} x_{j}} \psi= & -\delta^{-2} \frac{\left(x-\hat{z}_{r_{0}}\right)_{i}}{\left|x-\hat{z}_{r_{0}}\right|} \frac{\left(x-\hat{z}_{r_{0}}\right)_{j}}{\left|x-\hat{z}_{r_{0}}\right|} \\
& +\delta^{-1} \frac{\delta_{i j}}{\left|x-\hat{z}_{r_{0}}\right|}-\delta^{-1} \frac{\left(x-\hat{z}_{r_{0}}\right)_{i}\left(x-\hat{z}_{r_{0}}\right)_{j}}{\left|x-\hat{z}_{r_{0}}\right|^{3}}
\end{aligned}
$$

Let

$$
\begin{aligned}
A:= & -1+\delta^{-2} \partial_{\eta_{j}} a_{i}(D \psi)\left(\frac{\left(x-\hat{z}_{r_{0}}\right)_{i}}{\left|x-\hat{z}_{r_{0}}\right|} \frac{\left(x-\hat{z}_{r_{0}}\right)_{j}}{\left|x-\hat{z}_{r_{0}}\right|}\right) \\
& +\delta^{-1} \partial_{\eta_{j}} a_{i}(D \psi)\left(\frac{\left(x-\hat{z}_{r_{0}}\right)_{i}\left(x-\hat{z}_{r_{0}}\right)_{j}}{\left|x-\hat{z}_{r_{0}}\right|^{3}}-\frac{\delta_{i j}}{\left|x-\hat{z}_{r_{0}}\right|}\right) .
\end{aligned}
$$

Then using (3.11) and the notation in (3.12) we see that

$$
(C \eta)^{-1}\left(\partial_{t} \psi-\partial_{\eta_{j}} a_{i}(D \psi) \partial_{x_{i} x_{j}} \psi\right)=A
$$

and we are left with the task to prove that $A$ can be constructed to be nonnegative. Using the assumption in (1.2) we see that

$$
A \geq-1+\delta^{-2} \nu|D \psi|^{p-2}+\delta^{-1}|D \psi|^{p-2} \frac{(\nu-L)}{\left|x-\hat{z}_{r_{0}}\right|} .
$$

Next, since $\left|x-\hat{z}_{r_{0}}\right| \geq r_{0}$ in $D_{\delta}$ we can conclude, recalling $L \geq \nu>0$, that

$$
A \geq-1+\delta^{-1}\left(\delta^{-1} \nu-L / r_{0}\right)|D \psi|^{p-2} .
$$

Using that $|D \psi|=\delta^{-1} C \eta$ we see from the last display that

$$
A \geq-1+\delta^{-1}\left(\delta^{-1} \nu-L / r_{0}\right)\left(\delta^{-1} C \eta\right)^{p-2} .
$$

We now restrict $\delta$ so that $\delta^{-1}$ is larger than $2 L /\left(\nu r_{0}\right)$. Consequently, we obtain

$$
A \geq-1+\frac{1}{2} \delta^{-p}(C \eta)^{p-2}
$$

Using (3.17), together with the facts that $p<2$ and $\eta \leq 1$ in $D_{\delta}$, we can conclude that

$$
A \geq-1+\frac{1}{2}(1-\exp (-1))^{2-p} \delta^{-p}
$$


Hence, if we let $\delta=\min \left\{r_{0} / 10, \nu r_{0} /(2 L), 4^{-1 / p}\right\}$, then $A \geq 0$. In particular, we can conclude the validity of (3.6). We now apply $(3.6)$ with $(x, t) \equiv(z, \tau)$ and, appealing to the elementary inequalities

$$
(1-\exp (-1)) s \leq 1-\exp (-s) \leq s \quad \forall s \in[0,1],
$$

we obtain

$$
\begin{aligned}
\tilde{u}(z, \tau) & \leq \psi(z, \tau) \\
& =C\left(1-\exp \left(-\delta^{-1} \operatorname{dist}(z, \partial \Omega)\right)\right) \\
& \leq C \delta^{-1} \operatorname{dist}(z, \partial \Omega) .
\end{aligned}
$$

This allows us to conclude the proof of the right hand side inequality in (3.1) after scaling back to the original solution $u$ and applying (1.20).

Focusing then on the proof of the inequality on the left hand side in (3.1), we consider $\tilde{u}(x, t)=u\left(x, t \theta_{u}^{2-p}\right) / \theta_{u}$ for all $(x, t)$ such that $\left(x, t \theta_{u}^{2-p}\right) \in Q_{4, \phi}^{\theta_{u},+}(0,0)$, i.e., for $(x, t) \in Q_{4, \phi}^{1,+}(0,0)$. We then have that

$$
\tilde{u}\left(e_{n}, 0\right)=1 .
$$

Using the Harnack inequality outlined in (1.6)-(1.8) we see, also using (3.20), that there exists $\kappa \equiv \kappa(n, p, \nu, L), 0<\kappa<1$, and $c_{2} \equiv c_{2}(n, p, \nu, L), 1<c_{2}<\infty$, such that

$$
c_{2}^{-1} \leq \inf _{t \in\left(-4 \kappa^{2}, 4 \kappa^{2}\right)} \tilde{u}\left(e_{n}, t\right) \leq \sup _{t \in\left(-4 \kappa^{2}, 4 \kappa^{2}\right)} \tilde{u}\left(e_{n}, t\right) \leq c_{2} .
$$

We let $\delta \in\left(0, r_{0} / 10\right]$. Consider $(z, \tau) \in\left(\Omega_{\delta} \cap B\left(0, r_{0}\right)\right) \times\left(-\kappa^{2}, \kappa^{2}\right) \subset Q_{\kappa, \phi}^{2,+}(0,0)$, where $\Omega_{\delta}$ is defined in (3.3). Given $z$, we again let $\hat{z} \in \partial \Omega$ be such that $\mid z-$ $\hat{z} \mid=\operatorname{dist}(z, \partial \Omega)$ and we use (1.11) to find $\hat{z}^{r_{0}} \in \Omega$ such that $B\left(\hat{z}^{r_{0}}, r_{0}\right) \subset \Omega$ and $\overline{B\left(\hat{z}^{r_{0}}, r_{0}\right)} \cap \partial \Omega=\{\hat{z}\}$. Since the normal vector of the surface $\left\{x_{n}=\phi\left(x^{\prime}\right)\right\}$ is continuous, we necessarily have that $z-\hat{z}$ and $\hat{z}^{r_{0}}-\hat{z}$ are parallel and consequently, due to the orientation,

$$
r_{0}>\left|z-\hat{z}^{r_{0}}\right|=r_{0}-\operatorname{dist}(z, \partial \Omega)>r_{0}-\delta .
$$

Given $\tau \in\left(-\kappa^{2}, \kappa^{2}\right)$, we also let $I_{\kappa}(\tau)=\left(\tau-\kappa^{2}, \tau\right]$. Furthermore, we set

$$
m_{\delta}:=\inf _{E^{\delta} \times\left(-2 \kappa^{2}, 2 \kappa^{2}\right)} \tilde{u}(x, t),
$$

where $E^{\delta}:=\Omega \cap(-1,1)^{n} \cap\{x \in \Omega: \operatorname{dist}(x, \partial \Omega) \geq \delta\},(-1,1)^{n}$ is the $n$-dimensional cube $(-1,1) \times \cdots \times(-1,1)$. With $(z, \tau)$ fixed, we construct, following the proof of [29, Proposition 4.2], a barrier as follows. We let

$$
\begin{aligned}
& \tilde{\psi}(x, t):=\exp (-1) m_{\delta}(\tilde{\eta}(x, t)-1), \\
& \tilde{\eta}(x, t):=\exp \left(-\delta^{-1}\left(\left|x-\hat{z}^{r_{0}}\right|-r_{0}\right)\right) \exp \left((t-\tau) / \kappa^{2}\right) .
\end{aligned}
$$

In this case we consider the set

$$
\tilde{D}_{\delta}:=\left\{x \in \Omega: r_{0}-\delta<\left|x-\hat{z}^{r_{0}}\right|<r_{0}\right\} \times I_{\kappa}(\tau) .
$$

In particular, by (3.22) we have that $z \in \tilde{D}_{\delta}$. We now want to prove that $\delta$ can be chosen so that $\tilde{\psi}$ satisfies

$$
\tilde{\psi}(x, t) \leq \tilde{u}(x, t) \text { whenever }(x, t) \in \tilde{D}_{\delta} .
$$


Again, using the comparison principle we see that to achieve this it is enough to prove that

$$
\begin{array}{ll}
\tilde{\psi}(x, t) \leq \tilde{u}(x, t) \quad & \text { on } \partial_{P} \tilde{D}_{\delta}, \\
\partial_{t} \tilde{\psi}-\operatorname{div} a(D \tilde{\psi}) \leq 0 & \text { in } \tilde{D}_{\delta} .
\end{array}
$$

To obtain (3.26), note first that if $(x, t) \in\left\{x \in \Omega:\left|x-\hat{z}^{r_{0}}\right|=r_{0}\right\} \times I_{\kappa}(\tau)$, then $\tilde{\psi}(x, t) \leq 0$, and for $(x, t) \in\left\{x \in \Omega:\left|x-\hat{z}^{r_{0}}\right|=r_{0}-\delta\right\} \times I_{\kappa}(\tau)$ we have that $\tilde{\psi}(x, t) \leq(1-\exp (-1)) m_{\delta} \leq \tilde{u}(x, t)$. Furthermore, if $(x, t) \in\left\{x \in \Omega: r_{0}-\delta<\right.$ $\left.\left|x-\hat{z}^{r_{0}}\right|<r_{0}\right\} \times\left\{t: t=\tau-\kappa^{2}\right\}$, then we see that $\tilde{\psi}(x, t) \leq 0$. Hence, it only remains to prove that we can choose $\delta \equiv \delta\left(p, \nu, L, r_{0}, \kappa\right), 0<\delta \ll 1$, small enough so that we can prove (3.27). However, this can be verified by a direct calculation along the lines of the corresponding argument used to prove the right hand side inequality in (3.1) and we omit the details. Applying (3.25) to $(x, t)=(z, \tau)$ and using (3.19) and (3.22) we see that

$$
\tilde{u}(z, \tau) \geq \tilde{\psi}(z, \tau) \geq \exp (-1)(1-\exp (-1)) m_{\delta} \operatorname{dist}(z, \partial \Omega) .
$$

To complete the proof it now only remains to use (3.21) to bound $m_{\delta}$ from below with a constant depending only on $n, p, \nu, L, r_{0}$. For this we assume the contrary, i.e., we assume that there is a point

$$
\left(\tilde{z}_{0}, \tilde{t}_{0}\right) \in E^{\delta} \times\left(-2 \kappa^{2}, 2 \kappa^{2}\right) \text { such that } \tilde{u}\left(\tilde{z}_{0}, \tilde{t}\right) \leq \varepsilon
$$

for some $\varepsilon \in(0,1)$ to be chosen suitably small. We show that an elliptic Harnack chain can be constructed, similar to the case of the Laplace equation (see for instance [12]), to obtain a contradiction with (3.21). To this end, take $\varrho=\delta / 10$ and let $k$ be the smallest integer such that $k \geq 4^{n+1} 10 / \delta$. We may choose $\left(z^{j}\right)_{j=0}^{k+1}$ so that $\left|z^{j+1}-z^{j}\right|<\varrho / 2$, dist $\left(z^{j}, \partial \Omega\right) \geq 10 \varrho$ for all $j \in\{0,1, \ldots, k\}$, and so that $z^{k+1}=e_{n}$. Using the elliptic Harnack inequality as outlined in (1.6)-(1.8), together with (1.15) and (1.11), we find that $u\left(e_{n}, \tilde{t}\right) \leq \varepsilon c_{2}^{k+1}=c_{2}^{-2}<c_{2}^{-1}<1$ provided that we choose $\varepsilon:=c_{2}^{-k-3}$. Chosen this way $\varepsilon$ depends only on $n, p, \nu, L, r_{0}$. An important feature in the construction is that $u\left(z^{j}, \tilde{t}\right) \leq 1$ for all $j \in\{0,1, \ldots, k\}$ and hence also $u\left(z^{j}, \tilde{t}\right)^{2-p} \leq 1$. Thus we obtain a contradiction with (3.21) and the proof is complete.

Remark 3.2. Note that the inequality on the right hand side in (3.1) in Lemma 3.1 could also be proved by referring to the gradient estimate in Lemma 2.2. However, we have chosen to present a barrier type argument.

3.1. The final argument. Let $u$ be as in Theorem 1.1 and assume also that (2.9) holds. Define

$$
\tilde{u}(x, t)=u\left(x, t \Lambda_{u}^{2-p}\right) / \Lambda_{u}, \tilde{v}(x, t)=v\left(x, t \Lambda_{v}^{2-p}\right) / \Lambda_{v}
$$

whenever $(x, t) \in Q_{4, \phi}^{1,+}(0,0)$. Then

$$
\sup _{Q_{4, \phi}^{1,+}(0,0)} \tilde{u}=1=\sup _{Q_{4, \phi}^{1,+}(0,0)} \tilde{v} .
$$

Using Lemma 2.2 we see that $D \tilde{w}$ exists and is continuous up to $\Delta_{2, \phi}^{1}(0,0)$ for $\tilde{w} \in\{\tilde{u}, \tilde{v}\}$ and that there exist $\rho=\rho(n, p, \nu, L), 0<\rho \leq 1, c=c(n, p, \nu, L)$, $1 \leq c<\infty$, and $\sigma=\sigma(n, p, \nu, L), 0<\sigma \leq 1$ such that

$$
|D \tilde{w}(x, t)-D \tilde{w}(y, s)| \leq c\left(|x-y|+|t-s|^{1 / p}\right)^{\sigma}
$$


holds whenever $(x, t),(y, s) \in Q_{\rho, \phi}^{1,+}(0,0)$ and for $\tilde{w} \in\{\tilde{u}, \tilde{v}\}$. In the following we let $h$ be the function $h(x, t)=h\left(x^{\prime}, x_{n}, t\right)=x_{n}-\phi\left(x^{\prime}\right)$. Consider $(x, t),(y, s) \in Q_{\rho, \phi}^{1,+}(0,0)$ and $\tilde{w} \in\{\tilde{u}, \tilde{v}\}$. Then, using the fact that $\tilde{w}\left(x^{\prime}, \phi\left(x^{\prime}\right), t\right)=0$ for $\left(x^{\prime}, \phi\left(x^{\prime}\right), t\right) \in$ $\Delta_{2, \phi}^{1}(0,0)$, we see, using the fundamental theorem of calculus, that

$$
\frac{\tilde{w}(x, t)}{h(x, t)}=\int_{0}^{1} \tilde{w}_{x_{n}}\left(x^{\prime}, \tau x_{n}+(1-\tau) \phi\left(x^{\prime}\right), t\right) d \tau .
$$

Hence, using (3.30), the last display and (1.10), we can conclude that

$$
\left|\frac{\tilde{w}(x, t)}{h(x, t)}-\frac{\tilde{w}(y, s)}{h(y, s)}\right| \leq c\left(|x-y|+|t-s|^{1 / p}\right)^{\sigma} .
$$

Using (3.32), for $\tilde{u}$ and $\tilde{v}$, and scaling and translating back, we see that

$$
\left|\frac{u(x, t)}{h(x, t)}-\frac{u(y, s)}{h(y, s)}\right| \leq \hat{c} \frac{\Lambda_{u}}{r}\left(\frac{|x-y|}{r}+\left(\frac{1}{\Lambda_{u}}\right)^{(2 / p-1)}\left(\frac{|s-t|}{r^{p}}\right)^{1 / p}\right)^{\sigma}
$$

whenever $(x, t),(y, s) \in Q_{\rho r, \phi}^{\Lambda_{u},+}\left(x_{0}, t_{0}\right)$ and that

$$
\left|\frac{v(x, t)}{h(x, t)}-\frac{v(y, s)}{h(y, s)}\right| \leq \hat{c} \frac{\Lambda_{v}}{r}\left(\frac{|x-y|}{r}+\left(\frac{1}{\Lambda_{v}}\right)^{(2 / p-1)}\left(\frac{|s-t|}{r^{p}}\right)^{1 / p}\right)^{\sigma}
$$

whenever $(x, t),(y, s) \in Q_{\rho r, \phi}^{\Lambda_{v},+}\left(x_{0}, t_{0}\right)$. Furthermore, using Lemma 3.1 we see that that there exist constants $c_{1}=c_{1}(n, p, \nu, L), 1 \leq c_{1}<\infty, \varrho_{0} \equiv \varrho_{0}\left(n, p, \nu, L, r_{0}\right)$, $0<\varrho_{0}<1$, such that

$$
c_{1}^{-1} \theta_{u}\left(x_{n}-\phi\left(x^{\prime}\right)\right) \leq u(x, t) \leq c_{1} \lambda_{u} \theta_{u}\left(x_{n}-\phi\left(x^{\prime}\right)\right)
$$

whenever $(x, t) \in\left(\Omega \cap\left(B^{\prime}\left(0, r_{0}\right) \times[-1,1]\right)\right) \times\left(-\theta_{u}^{2-p} \varrho_{0}^{p}, \theta_{u}^{2-p} \varrho_{0}^{p}\right)$ and

$$
c_{1}^{-1} \theta_{v}\left(x_{n}-\phi\left(x^{\prime}\right)\right) \leq v(x, t) \leq c_{1} \lambda_{v} \theta_{v}\left(x_{n}-\phi\left(x^{\prime}\right)\right)
$$

whenever $(x, t) \in\left(\Omega \cap\left(B^{\prime}\left(0, r_{0}\right) \times[-1,1]\right)\right) \times\left(-\theta_{v}^{2-p} \varrho_{0}^{p}, \theta_{v}^{2-p} \varrho_{0}^{p}\right)$. Theorem 1.1 is now a consequence of (3.33) - (3.36) and the identity

$$
\begin{aligned}
\left(\frac{u(x, t)}{v(x, t)}-\frac{u(y, s)}{v(y, s)}\right)= & \frac{h(x, t)}{v(x, t)}\left(\frac{u(x, t)}{h(x, t)}-\frac{u(y, s)}{h(y, s)}\right) \\
& +\frac{u(y, s)}{h(y, s)} \frac{h(x, t)}{v(x, t)} \frac{h(y, s)}{v(y, s)}\left(\frac{v(y, s)}{h(y, s)}-\frac{v(x, t)}{h(x, t)}\right) .
\end{aligned}
$$

\section{ACKNOWLEDGEMENTS}

This research was supported by the ERC grant 207573 "Vectorial Problems" and by the Academy of Finland project "Potential estimates and applications for nonlinear parabolic partial differential equations".

\section{REFERENCES}

[1] Emilio Acerbi and Giuseppe Mingione, Gradient estimates for a class of parabolic systems, Duke Math. J. 136 (2007), no. 2, 285-320, DOI 10.1215/S0012-7094-07-13623-8. MR.2286632 (2007k:35211)

[2] Hiroaki Aikawa, Tero Kilpeläinen, Nageswari Shanmugalingam, and Xiao Zhong, Boundary Harnack principle for p-harmonic functions in smooth Euclidean domains, Potential Anal. 26 (2007), no. 3, 281-301, DOI 10.1007/s11118-006-9036-y. MR2286038(2008a:31012) 
[3] B. Avelin, U. Gianazza and S. Salsa, Boundary estimates for certain degenerate and singular parabolic equations, Preprint (2012).

[4] Emmanuele DiBenedetto, Degenerate parabolic equations, Universitext, Springer-Verlag, New York, 1993. MR 1230384 (94h:35130)

[5] Emmanuele DiBenedetto and Avner Friedman, Hölder estimates for nonlinear degenerate parabolic systems, J. Reine Angew. Math. 357 (1985), 1-22, DOI 10.1515/crll.1985.357.1. MR783531 (87f:35134a)

[6] Emmanuele DiBenedetto, Ugo Gianazza, and Vincenzo Vespri, Harnack estimates for quasilinear degenerate parabolic differential equations, Acta Math. 200 (2008), no. 2, 181-209, DOI 10.1007/s11511-008-0026-3. MR2413134 (2009g:35130)

[7] Emmanuele DiBenedetto, Ugo Gianazza, and Vincenzo Vespri, Forward, backward and elliptic Harnack inequalities for non-negative solutions to certain singular parabolic partial differential equations, Ann. Sc. Norm. Super. Pisa Cl. Sci. (5) 9 (2010), no. 2, 385-422. MR2731161 (2011j:35121)

[8] Eugene B. Fabes, Nicola Garofalo, and Sandro Salsa, A backward Harnack inequality and Fatou theorem for nonnegative solutions of parabolic equations, Illinois J. Math. 30 (1986), no. 4, 536-565. MR857210 (88d:35089)

[9] E. B. Fabes and M. V. Safonov, Behavior near the boundary of positive solutions of second order parabolic equations, Proceedings of the conference dedicated to Professor Miguel de Guzmán (El Escorial, 1996), Special Issue, 1997, pp. 871-882, DOI 10.1007/BF02656492. MR 1600211 (99d:35071)

[10] E. B. Fabes, M. V. Safonov, and Yu Yuan, Behavior near the boundary of positive solutions of second order parabolic equations. II, Trans. Amer. Math. Soc. 351 (1999), no. 12, 4947-4961, DOI 10.1090/S0002-9947-99-02487-3. MR1665328 (2000c:35085)

[11] Nicola Garofalo, Second order parabolic equations in nonvariational forms: boundary Harnack principle and comparison theorems for nonnegative solutions, Ann. Mat. Pura Appl. (4) 138 (1984), 267-296, DOI 10.1007/BF01762548. MR779547 (87f:35115)

[12] David S. Jerison and Carlos E. Kenig, Boundary behavior of harmonic functions in nontangentially accessible domains, Adv. in Math. 46 (1982), no. 1, 80-147, DOI 10.1016/00018708(82)90055-X. MR676988(84d:31005b)

[13] T. Kilpeläinen and P. Lindqvist, On the Dirichlet boundary value problem for a degenerate parabolic equation, SIAM J. Math. Anal. 27 (1996), no. 3, 661-683, DOI 10.1137/0527036. MR $1382827(97 \mathrm{~b}: 35118)$

[14] Juha Kinnunen and John L. Lewis, Higher integrability for parabolic systems of p-Laplacian type, Duke Math. J. 102 (2000), no. 2, 253-271, DOI 10.1215/S0012-7094-00-10223-2. MR:1749438(2001b:35152)

[15] Tuomo Kuusi and Giuseppe Mingione, Potential estimates and gradient boundedness for nonlinear parabolic systems, Rev. Mat. Iberoam. 28 (2012), no. 2, 535-576. MR2916967

[16] T. Kuusi and G. Mingione, Gradient regularity for nonlinear parabolic equations, Ann. Sc. Norm. Super. Pisa Cl. Sci. (V) 12 (2013), 755-822.

[17] Tuomo Kuusi, Giuseppe Mingione, and Kaj Nyström, Sharp regularity for evolutionary obstacle problems, interpolative geometries and removable sets, J. Math. Pures Appl. (9) 101 (2014), no. 2, 119-151, DOI 10.1016/j.matpur.2013.03.004. MR3158698

[18] John L. Lewis and Kaj Nyström, Boundary behaviour for $p$ harmonic functions in Lipschitz and starlike Lipschitz ring domains (English, with English and French summaries), Ann. Sci. École Norm. Sup. (4) 40 (2007), no. 5, 765-813, DOI 10.1016/j.ansens.2007.09.001. MR2382861(2009d:35093)

[19] John Lewis and Kaj Nyström, Boundary behavior and the Martin boundary problem for $p$ harmonic functions in Lipschitz domains, Ann. of Math. (2) 172 (2010), no. 3, 1907-1948, DOI 10.4007/annals.2010.172.1907. MR2726103 (2011k:31008)

[20] John L. Lewis and Kaj Nyström, Regularity and free boundary regularity for the p Laplacian in Lipschitz and $C^{1}$ domains, Ann. Acad. Sci. Fenn. Math. 33 (2008), no. 2, 523-548. MR2431379(2010b:35500)

[21] John L. Lewis and Kaj Nyström, Boundary behaviour of p-harmonic functions in domains beyond Lipschitz domains, Adv. Calc. Var. 1 (2008), no. 2, 133-170, DOI 10.1515/ACV.2008.005. MR.2427450 (2009i:35111) 
[22] John L. Lewis and Kaj Nyström, Regularity of Lipschitz free boundaries in two-phase problems for the p-Laplace operator, Adv. Math. 225 (2010), no. 5, 2565-2597, DOI 10.1016/j.aim.2010.05.005. MR.2680176 (2012c:35486)

[23] John L. Lewis and Kaj Nyström, Regularity of flat free boundaries in two-phase problems for the p-Laplace operator, Ann. Inst. H. Poincaré Anal. Non Linéaire 29 (2012), no. 1, 83-108, DOI 10.1016/j.anihpc.2011.09.002. MR2876248

[24] John L. Lewis and Kaj Nyström, Regularity and free boundary regularity for the p-Laplace operator in Reifenberg flat and Ahlfors regular domains, J. Amer. Math. Soc. 25 (2012), no. 3, 827-862, DOI 10.1090/S0894-0347-2011-00726-1. MR2904575

[25] Gary M. Lieberman, Boundary and initial regularity for solutions of degenerate parabolic equations, Nonlinear Anal. 20 (1993), no. 5, 551-569, DOI 10.1016/0362-546X(93)90038-T. MR $1207530(94 \mathrm{e}: 35041)$

[26] Kaj Nyström, The Dirichlet problem for second order parabolic operators, Indiana Univ. Math. J. 46 (1997), no. 1, 183-245, DOI 10.1512/iumj.1997.46.1277. MR.1462802 (98k:35087)

[27] Sandro Salsa, Some properties of nonnegative solutions of parabolic differential operators (English, with Italian summary), Ann. Mat. Pura Appl. (4) 128 (1981), 193-206, DOI 10.1007/BF01789473. MR640782 (83j:35078)

[28] Mikhail V. Safonov and Yu Yuan, Doubling properties for second order parabolic equations, Ann. of Math. (2) 150 (1999), no. 1, 313-327, DOI 10.2307/121104. MR1715327 (2000h:35059)

[29] Giuseppe Savaré and Vincenzo Vespri, The asymptotic profile of solutions of a class of doubly nonlinear equations, Nonlinear Anal. 22 (1994), no. 12, 1553-1565, DOI 10.1016/0362546X(94)90188-0. MR1285092 (96a:35084)

Aalto University, Institute of Mathematics, P.O. Box 11100, Fi-00076 Aalto, Finland

E-mail address: tuomo.kuusi@aalto.fi

Dipartimento di Matematica e Informatica, Università di Parma, Parco Area delle Scienze 53/A, Campus, 43124 Parma, Italy

E-mail address: giuseppe.mingione@unipr.it

Department of Mathematics, Uppsala University, S-751 06 Uppsala, Sweden

E-mail address: kaj.nystrom@math.uu.se 